\title{
Raltegravir in combination with other antiretroviral agents for the treatment of HIV infection
}

This article was published in the following Dove Press journal:

Infection and Drug Resistance

24 April 2010

Number of times this article has been viewed

\author{
Lisa M Chirch \\ Roy T Steigbigel \\ Division of Infectious Diseases, \\ Department of Medicine, Stony Brook \\ University Medical Center, Stony \\ Brook, New York, USA
}

\begin{abstract}
Raltegravir, an inhibitor of the HIV-1 integrase enzyme, is the first available agent in a new class of antiretroviral drugs. Raltegravir has been studied extensively in clinical trials, and has been well tolerated and highly effective in both treatment-naïve and -experienced patients. Resistance to raltegravir is unusual given its recent availability, but resistance with identified viral mutation pathways in the integrase gene in patients receiving the drug does occur.
\end{abstract}

Keywords: raltegravir, integrase, human immunodeficiency virus, drug resistance

\section{Introduction}

Recent years have witnessed dramatic changes in the management of patients infected with human immunodeficiency virus (HIV). International guidelines have been updated, and experts are now recommending beginning antiretroviral therapy (ART) earlier in the course of infection. ${ }^{1}$ Data from several large international cohort studies indicate that unchecked viremia and delayed therapy not only increase the risk of opportunistic infection, but also the incidence of cardiovascular disease, renal disease, malignancy, and overall mortality. ${ }^{2,3}$ Recent guidelines recommend that clinicians obtain resistance testing at baseline, given the problem of transmitted viral resistance. The prevalence of transmitted resistance is variable, and is estimated to occur in as many as $10 \%$ of new infections. ${ }^{4}$ Increasing numbers of previously treated patients harbor virus resistant to drugs in each of the three original classes of antiretrovirals: the nucleoside reverse transcriptase inhibitors (NRTI), non-NRTIs, and protease inhibitors (PI). To help overcome these hurdles, several new fixed-dose combination (FDC) regimens and drugs with entirely new mechanisms of action are now available: the CCR5-coreceptor inhibitors and the integrase (IN) inhibitors.

Raltegravir (Isentress ${ }^{\circledR}$; Merck and Co., Inc., Whitehouse Station, NJ, USA), an inhibitor of the HIV-1 IN enzyme, is the first agent in a new class of antiretroviral drugs. Initially approved in 2007 for the treatment of patients infected with multi-drug resistant HIV, raltegravir was recently also approved by the US Food and Drug Administration (FDA) for ART-naïve patients. Raltegravir has been studied extensively in clinical trials and has been shown to be highly effective and well tolerated. Resistance to raltegravir is still unusual given its recent availability, but mutation pathways in the IN gene that develop in patients receiving the drug has been observed. ${ }^{5}$ In this review recent developments in clinical research involving raltegravir and its role in the management of treatment-naïve and -experienced HIV-infected patients are discussed.
Correspondence: Lisa Chirch Health Sciences Center TI5-080, Stony Brook, New York I I794-8I 53, USA

$\mathrm{Tel}+\mathrm{I}$ (63I) 444-3490

Fax +I (63I) 444-75I 8

Email Ichirch@notes.cc.sunysb.edu 


\section{Pharmacology, mode of action, and pharmacokinetics}

HIV integrases are versatile transferases able to remove and transfer portions of double-stranded polyribonucleotide substrate to another gene location. ${ }^{6}$ In practical terms, following reverse transcription, HIV IN allows proviral DNA to integrate into host cell DNA, leading to transcription and production of new infectious virions.?

IN is a 288 -amino acid protein encoded along with protease and reverse transcriptase by the pol gene. It has three independent domains, serving as three potential drug targets: the N-terminal domain, the central or catalytic domain, and the C-terminal domain. IN binds each end of viral DNA and catalyzes endonucleotide cleavage from each end ( 3 ' processing). The cleaved DNA forms the pre-integration complex, followed by strand transfer with joining of viral and cellular DNA (integration). ${ }^{7,8}$ Raltegravir is a strandtransfer inhibitor (INSTI), and blocks docking of the enzyme to target DNA, inhibiting viral integration. Although other potential targets within viral IN are under investigation, the strand-transfer inhibitors are the only agents to date that have progressed to phase III clinical trials. ${ }^{6}$

Pharmacokinetic studies demonstrate potent in vitro activity of raltegravir against HIV-1, with a 95\% inhibitory concentration $\left(\mathrm{IC}_{95}\right)$ of $33 \mathrm{nM}$ in $50 \%$ human serum. ${ }^{9}$ The drug is rapidly absorbed, with median time to peak plasma concentration ranging from 0.5 to 1.3 hours, and steady state is achieved within 2 days of multiple-dose administration. Overall pharmacokinetic parameters following a single dose of $400 \mathrm{mg}$ are similar in males and females. ${ }^{9}$

The approved clinical dose of raltegravir is $400 \mathrm{mg}$ orally twice daily. The drug is metabolized primarily via glucuronidation mediated by the uridine diphosphate glucuronosyl transferase 1A1 isoenzyme (UGT 1A1), leading to relatively few drug - drug interactions in comparison to agents metabolized primarily by cytochrome $\mathrm{P} 450$ enzymes. Inducers or inhibitors of UGT 1 A1 may have an effect on raltegravir concentrations. For example, two pharmacokinetic studies found that coadministration of rifampin, a UGT 1A1 inducer, resulted in lower plasma raltegravir concentrations; increasing the raltegravir dose to $800 \mathrm{mg}$ twice daily compensates for this effect on raltegravir exposure (increases the area under the curve [AUC]) but does not overcome the effect on trough concentrations. ${ }^{10}$ Coadministration of rifampin with raltegravir should thus be undertaken with caution. Tipranavir, a protease inhibitor coadministered with ritonavir used for the treatment of drug-resistant HIV-1, is also an inducer of UGT $1 \mathrm{~A} 1$.
Although raltegravir concentrations at 12 hours (C12) were decreased when coadministered with tipranavir in healthy subjects, other pharmacokinetic parameters were not substantially affected, and there were no differences in safety or efficacy profiles. ${ }^{11}$ Atazanavir, a protease inhibitor used frequently in the treatment of HIV-1, is an inhibitor of both cytochrome P450 3A and UGT 1A1. When coadministered with raltegravir to healthy subjects in multiple doses, atazanavir and atazanavir plus ritonavir modestly increased plasma levels of raltegravir. ${ }^{12}$ Raltegravir is more soluble at basic gastric $\mathrm{pH}$ levels, and coadministration of protonpump inhibitors, such as omeprazole, results in increased plasma concentrations of raltegravir (3- to 4-fold increase in AUC). ${ }^{13}$ No dosage adjustments are currently recommended when atazanavir, tipranavir, or omeprazole are administered with raltegravir.

Raltegravir is administered without regard to food. No clinically important pharmacokinetic differences were observed in subjects with severe renal impairment or mild to moderate hepatic impairment, so no dose adjustments are recommended in patients with these conditions. ${ }^{14}$

\section{Clinical efficacy: treatment-naïve patients}

Based on efficacy data in treatment-naïve patients from Protocol 004 and STARTMRK, raltegravir was approved for use in this patient population by the FDA in mid 2009. ${ }^{15-18}$ Part 1 of Protocol 004 randomized 35 ART-naïve patients to placebo or to raltegravir at four different doses $(100 \mathrm{mg}$, $200 \mathrm{mg}, 400 \mathrm{mg}$ or $600 \mathrm{mg}$ ) administered twice daily for ten days. In all cases, raltegravir resulted in dramatic reductions in HIV-1 RNA (approximately a $2.0 \log _{10}$ reduction), and at least $50 \%$ of patients receiving any dose of raltegravir achieved a viral load of $<400$ copies/mL by day $10 .{ }^{15}$ In part 2 of the protocol 198 ART-naïve patients with HIV-1 RNA levels of at least 5,000 copies $/ \mathrm{mL}$ and $\mathrm{CD} 4+$ cell counts of at least 100 cells $/ \mathrm{mm}^{3}$ were randomized to receive efavirenz $600 \mathrm{mg}$ or raltegravir (at one of the above four doses) with a nucleoside backbone of tenofovir $300 \mathrm{mg}$ and lamivudine $300 \mathrm{mg} .{ }^{16}$ All drugs were dosed daily, except for raltegravir, which was dosed twice daily, as in Part 1 . At 48 weeks all patients receiving raltegravir were stratified into one group receiving $400 \mathrm{mg}$ twice daily, to continue through 96 weeks. ${ }^{17}$ More than $90 \%$ of patients receiving any dose of raltegravir achieved HIV-1 RNA levels of $<400$ copies/mL by week 4 , and through week 8 more patients receiving raltegravir than efavirenz achieved HIV-1 RNA levels of $<50$ copies $/ \mathrm{mL}$. Thus, viral load reduction was more rapid in patients on 
raltegravir, although the proportion of patients in each group with virologic suppression to $<50$ copies $/ \mathrm{mL}$ was similar at 24 and 48 weeks. ${ }^{16}$ Three percent of patients in each group experienced virologic failure: 2 patients who had failed a raltegravir-based regimen were found to have the $\mathrm{N} 155 \mathrm{H}$ mutation. At week $96,83 \%$ of patients in the raltegravir group and $84 \%$ of patients in the efavirenz group had achieved HIV-1 RNA levels of $<50$ copies $/ \mathrm{mL} .{ }^{17}$ Patients in both groups had similar increases in CD4+ cell count (221 vs $232 \mathrm{cells} / \mathrm{mm}^{3}$, respectively). One additional patient in each group experienced virologic failure, although no integrase resistance mutations were identified.

STARTMRK is an ongoing international, randomized, double-blind, Phase III study comparing raltegravir $400 \mathrm{mg}$ twice daily to efavirenz $600 \mathrm{mg}$ nightly, both in combination with tenofovir $300 \mathrm{mg}$ and emtricitabine $200 \mathrm{mg}$ in FDC. ${ }^{18}$ Patients naïve to ART, aged 18 years or older, with HIV-1 RNA levels of $>5,000$ copies $/ \mathrm{mL}$ were eligible for study entry. Patients were excluded if they had renal insufficiency or decompensated hepatitis; patients with chronic hepatitis were eligible if their serum aminotransferase levels were less than 5 times the upper limit of normal. Pregnant or breastfeeding women were excluded, as were patients with documented genotypic resistance to tenofovir, emtricitabine, or efavirenz. Virologic failure was defined as either nonresponse (those who did not achieve a viral load of $<50$ copies $/ \mathrm{mL}$ at any stage during the study) or rebound (those who had a viral load of $>50$ copies $/ \mathrm{mL}$ on 2 consecutive measurements after an initial response to treatment).$^{18}$ Virologic failure was investigated by genotyping of the integrase coding sequence.

Eighty-six percent of patients in the raltegravir arm achieved a viral load of $<50$ copies $/ \mathrm{mL}$ at week 48 , compared with $81.9 \%$ in the efavirenz arm $(P<0.001$ for noninferiority). Of note, the time to achieve viral suppression was significantly shorter for patients on raltegravir than for those on efavirenz $(P<0.001)$. Although the exact mechanism is unknown, this rapid decay in viremia has been attributed to raltegravir's inhibition of reverse transcribed HIV DNA into the host genome, a step that occurs later in the viral life cycle. ${ }^{19}$ Additionally, patients receiving raltegravir had greater increases in CD4+ cell counts from baseline at week $48\left(189\right.$ cells $/ \mathrm{mm}^{3}$ vs 163 cells $/ \mathrm{mm}^{3}$ for efavirenz recipients $(P<0.02) .^{18}$

Ten percent of patients on raltegravir vs $14 \%$ on efavirenz failed to achieve HIV-1 RNA levels $<50$ copies $/ \mathrm{mL}$. Genotypic susceptibility testing was performed on 15 patients who had sufficient virus for amplification. Four of 8 patients on raltegravir had virus with integrase mutations (2 had
G140S, Q148H/R, 1 had Y143Y/H, 1 had Y143R), and 3 of the 7 patients on efavirenz had RT mutations (all 3 had K103N). ${ }^{18}$

Significantly more patients on efavirenz had drugrelated clinical adverse events, in particular, central nervous system (CNS)-related. The most common adverse events were dizziness (6\% raltegravir arm, 34\% efavirenz), headache ( $9 \%$ vs $14 \%$, respectively), and abnormal dreams ( $7 \%$ vs $13 \%$, respectively).

There have been no known cases to date of raltegravirresistant virus in newly infected individuals. The availability of raltegravir and other drugs in new classes may therefore prove particularly useful in such cases of transmitted resistance, for example, to nonnucleoside reverse transcriptase inhibitors, given the theoretical risk of suboptimal response to standard first-line regimens.

\section{Clinical efficacy: treatment- experienced patients}

Given its activity against HIV-1 with reverse transcriptase and/or protease mutations, raltegravir was initially approved in 2007, and is increasingly utilized for the treatment of multi-drug resistant virus. Its safety and efficacy in this setting have been demonstrated in multinational randomized Phase II and III clinical trials. In Protocol 005, patients with HIV-1 RNA levels of greater than 5000 copies/mL, CD4 cell counts above 50 cells $/ \mathrm{mm}^{3}$, and genotypic or phenotypic resistance to at least one NRTI, non-NRTI, and PI, received raltegravir or placebo in combination with optimized background therapy (OBT) as determined by the investigator. ${ }^{20}$

Patients in raltegravir arms received one of three doses: 200, 400, or $600 \mathrm{mg}$ twice daily. In both Phase II and III trials, genotypic and phenotypic sensitivity scores (GSS and PSS, respectively) represented the total number of active drugs in the OBT. The Protocol 005 patient population was highly treatment experienced, having received a median of 12 antiretroviral agents per patient, with a median of 4 drugs in the OBT. Thirty-six percent of patients were on enfuvirtide. At week 24 , patients receiving any dose of raltegravir achieved significantly greater degrees of viral suppression than did those receiving placebo with OBT (mean change in viral load from baseline was $-1.80 \log _{10}$ copies $/ \mathrm{mL}$ in the $200 \mathrm{mg}$ group, $-1.87 \log _{10}$ copies $/ \mathrm{mL}$ in the $400 \mathrm{mg}$ group, $-1.84 \log _{10}$ copies $/ \mathrm{mL}$ in the $600 \mathrm{mg}$ group, and $-0.35 \log _{10}$ copies $/ \mathrm{mL}$ for the placebo group, $P<0.001) .{ }^{20}$ Increases in CD4 counts were also significantly greater in all raltegravir groups compared to placebo, and treatment differences were consistent across all GSS and PSS. 
The BENCHMRK studies are ongoing randomized, double-blind, placebo-controlled Phase III trials similar in design to Phase II: BENCHMRK-1 in Europe, Asia, Australia, and Peru; BENCHMRK-2 in North and South America. 5,21 Patients were enrolled at a 2:1 ratio to receive either raltegravir $400 \mathrm{mg}$ twice daily or placebo, both with OBT. The most recent data from BENCHMRK are 96 week results presented at the Conference on Retroviruses and Opportunistic Infections (CROI) in February of 2009; the planned study duration is 256 weeks. ${ }^{22}$ As in Protocol 005, OBT was selected by the investigator based on previous treatment history and resistance testing, and results were stratified by GSS, PSS, and first use of darunavir or enfuvirtide. Patients were required to have an HIV-1 RNA level of greater than 1000 copies/mL and documented triple-class resistance on entry into the trial. The median CD4 counts and mean baseline viral loads were similar in the raltegravir and placebo-based groups (CD4 119 vs 123 cells $/ \mathrm{mm}^{3}$, respectively; and viral load 44,897 and 39,059 copies/mL, respectively). ${ }^{22}$ Twenty-five percent of patients in the raltegravir arm, and $28 \%$ of those on placebo had a GSS of zero.

Although the BENCHMRK patient population represents a highly treatment-experienced group with multi-drug resistant virus, 96-week data reveal sustained and potent viral load reduction and CD4 count reconstitution in patients on raltegravir plus OBT, in comparison with placebo (Figures 1 and 2). Fifty-seven percent of patients on raltegravir had HIV-1 RNA levels of $<50$ copies $/ \mathrm{mL}$ at 96 weeks, compared to $26 \%$ on placebo $(P<0.001$, adjusted for baseline viral load, and first use of enfuvirtide, darunavir, or active PI in OBT). Change from baseline CD4 cell count was also significantly greater in patients receiving raltegravir plus OBT compared to placebo (123 vs 49 cells $/ \mathrm{mm}^{3}$, respectively, $P<0.001) .^{22}$

Figure 3 depicts rates of viral suppression at week 96 stratified by GSS. As expected, patients with more active agents in their OBT had higher rates of virologic suppression in both the raltegravir and placebo arms (65\% vs 53\%, respectively, with a GSS of $\geq 2$ ). The most dramatic difference was observed between arms in patients who had no active agents in their OBT, or a GSS of 0 . These patients who received raltegravir had a rate of viral suppression to less than 50 copies $/ \mathrm{mL}$ at 96 weeks of $41 \%$, compared to $5 \%$ who received placebo plus OBT. ${ }^{22}$ Those who received either darunavir or enfuvirtide for the first time as part of their OBT also did significantly better than those who did not. The rates of virologic suppression in patients receiving both these agents in their OBT were similar between the two arms ( $79 \%$ on raltegravir, $63 \%$ on placebo), whereas patients with neither darunavir or enfuvirtide in their OBT did significantly better on raltegravir ( $56 \%$ suppressed to $<50$ copies $/ \mathrm{mL}$, compared to $19 \%$ on placebo).

Occurrence of AIDS-defining conditions (ADC) and death was examined at 48 weeks as a secondary endpoint

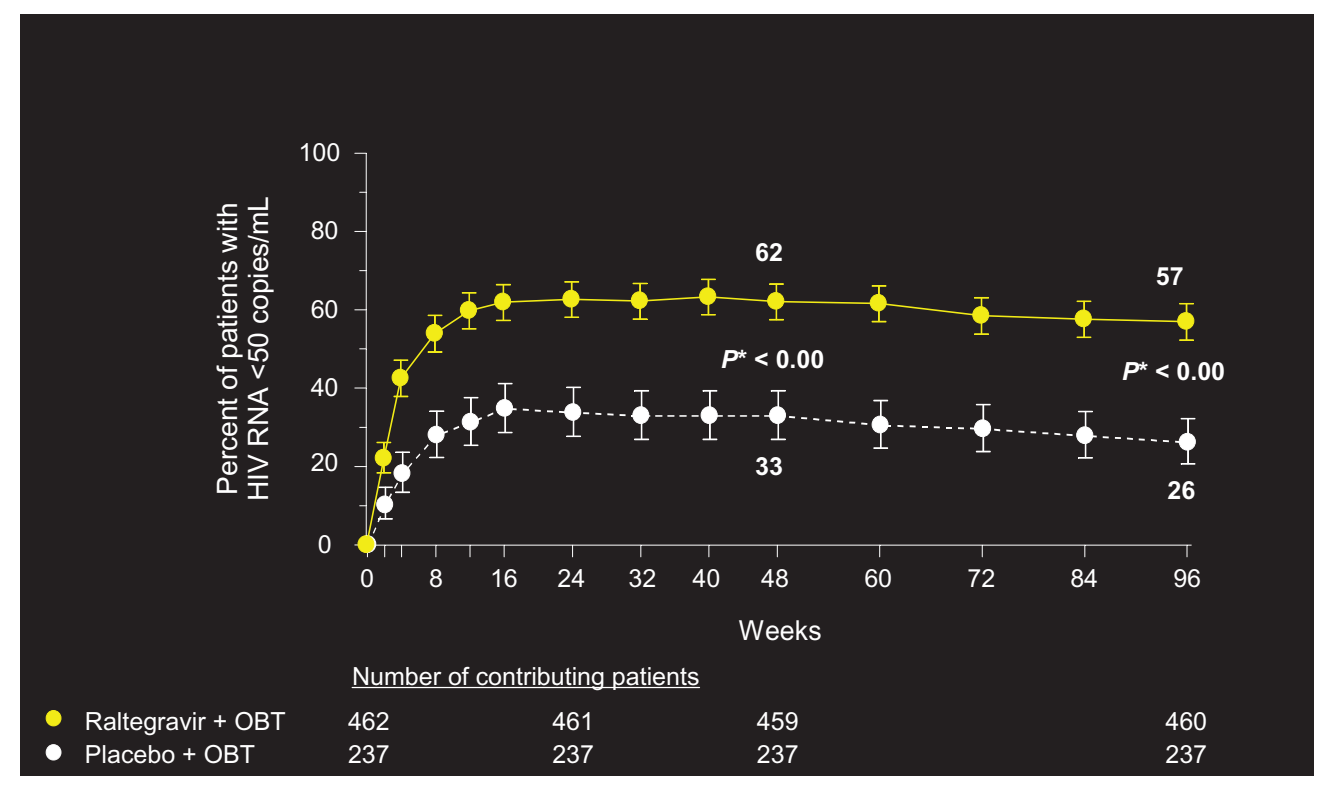

Figure I Percentage of patients $(95 \% \mathrm{Cl})$ achieving HIV RNA $<50$ copies $/ \mathrm{mL}$.

Copyright @ Merck Sharp \& Dohme Corp., a subsidiary of Merck \& Co., Inc., Whitehouse Station, NJ, USA.All Rights Reserved.

*P-value derived from a logistic regression model adjusted for baseline HIV RNA level $\left(\log _{10}\right)$, first enfuvirtide use in optimized background therapy (OBT), first darunavir use in $\mathrm{OBT}$, active protease inhibitors in OBT. 


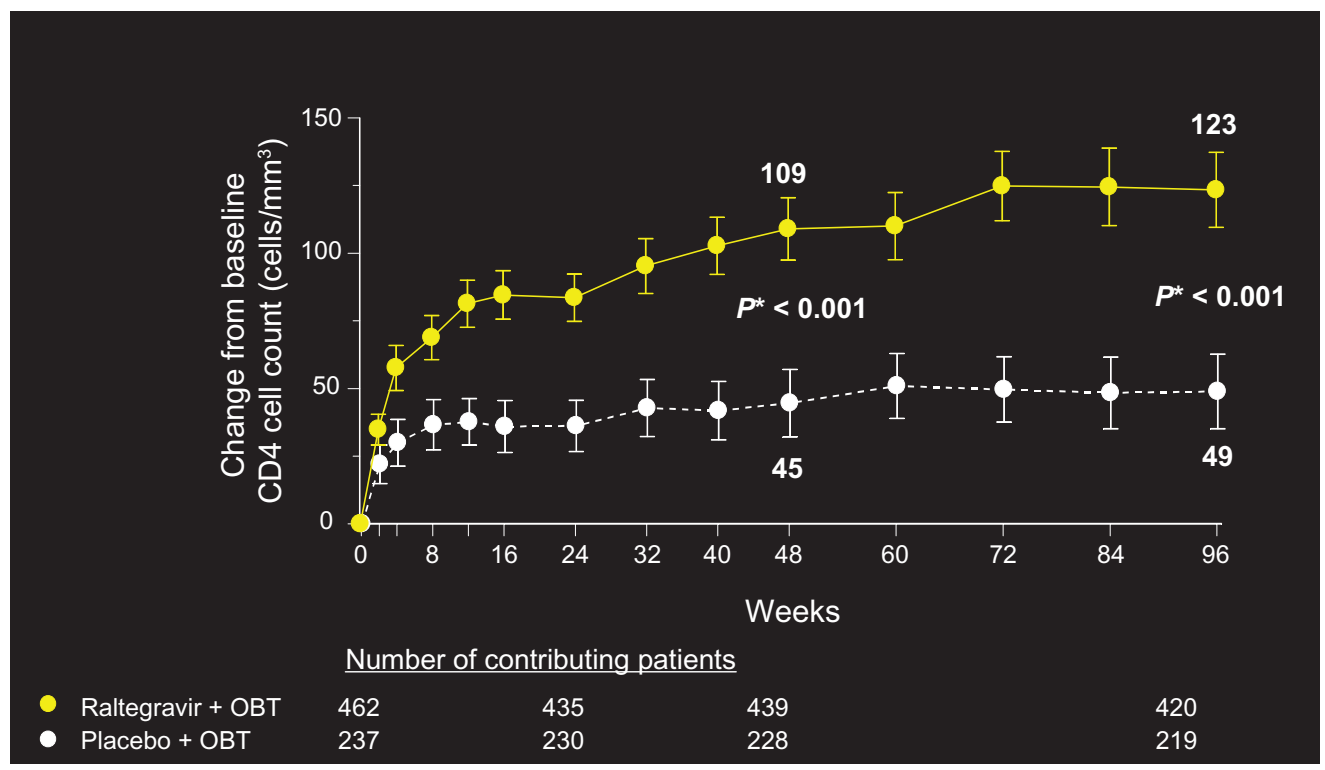

Figure 2 Change from baseline in CD4 cell count (cells $/ \mathrm{mm}^{3}$ ).

Copyright @ Merck Sharp \& Dohme Corp., a subsidiary of Merck \& Co., Inc., Whitehouse Station, NJ, USA. All Rights Reserved.

*P-value derived from mixed-effects model adjusted for: baseline CD4 count, stratum, treatment, visit, interactions between visit and previous variables.

Abbreviation: OBT, optimized background therapy.

in BENCHMRK. The median time to first new or recurrent ADC was 9.1 weeks in the raltegravir arm and 15 weeks in the placebo arm. The incidence rate of new ADCs or death was lower for patients on raltegravir (3.00 per 100 person-years [PYR] vs 6.78 per 100 PYR for placebo), but differences were not statistically significant. The most common ADC in both groups was esophageal candidiasis. ${ }^{23}$

Virologic failure was defined as failure to achieve HIV-1 RNA levels of $<400$ copies/mL or less than a $1 \log _{10}$ drop from baseline by week 16 . Patients were also considered to

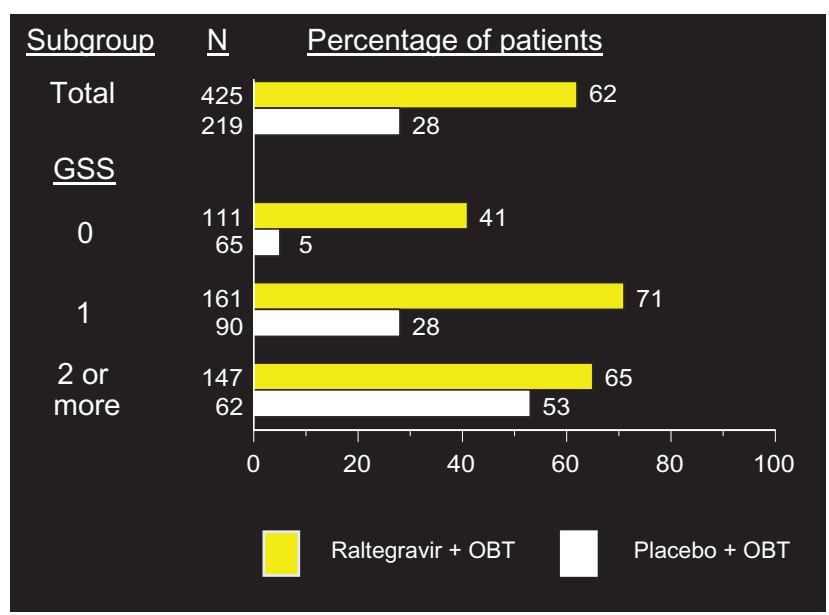

Figure 3 Percentage of patients with HIV RNA $<50$ copies $/ \mathrm{mL}$ at week 96 by genotypics sensitivity score (GSS). ${ }^{a}$

Copyright (c) Merck Sharp \& Dohme Corp., a subsidiary of Merck \& Co., Inc., Whitehouse Station, N.J., USA. All Rights Reserved.

*Virologic failures carried forward.

Abbreviation: OBT, optimized background therapy. have failed virologically if they experienced an increase in HIV-1 RNA of more than $1 \log _{10}$ copies/mL from the nadir level on 2 consecutive measurements, or 2 consecutive levels of greater than 400 copies/mL after having achieved a level of less than 400 copies/mL. ${ }^{21}$ Twenty-five patients had virologic failure between weeks 48 and 96: 17 of 370 on raltegravir plus OBT, 8 of 105 patients on placebo plus OBT. ${ }^{22}$ Of the 17 patients who failed on raltegravir, 11 had genotype analysis, and 7 had known raltegravir resistance mutations. Of note, three of these patients had a GSS of 0 , and one had a GSS of 1 . The most common major IN mutation was the $\mathrm{N} 155 \mathrm{H}$, present in all but one of the above seven patients. Two patients had the Q148 (one Q148H, one Q148R), and one had the $\mathrm{Y} 143 \mathrm{C}$. A variety of minor mutations were present in the seven patients. ${ }^{22}$ These results are consistent with what is currently understood about the development of resistance to IN inhibitor therapy: failure on raltegravir generally occurs when no other fully active drugs are included in the OBT, and is represented most commonly by the $\mathrm{Q} 148 \mathrm{H} / \mathrm{K} / \mathrm{R}$ or $\mathrm{N} 155 \mathrm{H}$ major mutations in combination with a variety of minor mutations. Raltegravir failure is associated with IN mutations in one of these two genetic pathways, defined by at least two mutations, including a major mutation at either $\mathrm{Q} 148 / \mathrm{K} / \mathrm{R}$ or $\mathrm{N} 155 \mathrm{H}$ and at least one minor mutation. The most common pathway to IN inhibitor resistance is Q148H plus G140S. Major mutations reduce susceptibility and viral replication capacity, whereas minor mutations emerge later and enhance resistance while improving replication capacity. ${ }^{4}$ 
Based on data from Protocol 005 and BENCHMRK, when considering the use of raltegravir for treatment-experienced individuals, it is imperative to include at least one other fully active agent in a treatment regimen, as patients receiving functional monotherapy with raltegravir are at high risk for virologic failure due to the development of mutations in IN. Retrospective and in vitro analyses were performed based on 48 week resistance data: baseline and virologic failure samples were available for 94 of the 105 patients who failed on raltegravir plus OBT at that time point. ${ }^{24}$ The Q148 pathway was selected over time, and conferred a larger reduction in raltegravir susceptibility than the N155 pathway. Both pathways reduced replication capacity, but the effect of minor mutations on replication capacity varied depending on the primary mutation. Fransen and colleagues also reported the exclusive association of G140A/S with Q148 mutations, and E92Q with N155H mutations. ${ }^{25}$ In general, the addition of secondary mutations enhanced resistance to raltegravir, and in some cases partially restored replication capacity.

\section{Clinical efficacy: novel combinations and switch studies}

The availability of raltegravir has led clinicians to utilize novel strategies in managing complex patients. For example, many NRTIs, in particular the thymidine analogues, such as stavudine, didanosine, and zidovudine, are associated with longitudinal toxicities, such as lipoatrophy and peripheral neuropathy, leading to the consideration of "nucleoside-sparing" regimens. Before the advent of the IN inhibitor class this regimen could only have consisted of an NNRTI and a PI, or a dual PI regimen. Medications in both these classes have shown to cause considerable toxicity, including increase in LDL cholesterol. ${ }^{26}$ Preliminary data on the combination of an unboosted PI, atazanavir, with raltegravir for treatment-experienced patients, were presented at the International AIDS Society Conference in Cape Town, South Africa, in July $2009 .{ }^{27}$ This was a single-arm, open-label pilot study, in which 27 treatment-experienced patients who were NRTI intolerant or had demonstrated NRTI resistance were switched from their current regimen to raltegravir $400 \mathrm{mg}$ and atazanavir $200 \mathrm{mg}$ twice daily. Patients were ineligible for the study if they had previously received raltegravir or had documented PI resistance. Use of proton-pump inhibitors was not permitted. The primary endpoint of the study was HIV-1 viral load of less than 50 copies $/ \mathrm{mL}$ at week 48. Twenty-two patients were already on PI-containing ART, and a majority (22 of 27) switched regimens due to intolerance or toxicity. Only five patients changed regimens due to NRTI resistance. Ninety-three percent of patients achieved and maintained virologic suppression to less than 50 copies/mL by week 24: of the two patients who failed to achieve suppression, one had developed the N155H IN mutation. Both had detectable HIV-1 viral loads at baseline. The regimen was well tolerated, with no dose reductions or significant adverse events. An increase in serum bilirubin was noted in a majority of patients. There were no significant changes in liver or renal function, and serum lipids did not change significantly at week 24 . Mean serum creatine phosphokinase increased significantly from study inception to week 24 (127 to $148 \mathrm{IU} / \mathrm{L}, P<0.05$ ).

Although these results are promising, it is important to note the small size and nonrandomized, open-label nature of this study. Investigations of other INSTI-containing, NRTI-sparing regimens are ongoing, including raltegravir in combination with darunavir/ritonavir and lopinavir/ ritonavir. Results from controlled randomized trials comparing NRTI-containing vs NRTI-sparing regimens will help establish the role of these regimens in various patient populations.

Results from additional "switch" studies were recently presented, in which raltegravir was substituted for other agents in patients with undetectable viral loads due to toxicity and/or intolerance. ${ }^{28,29}$ The EASIER investigators randomized 170 patients with triple-class resistance or intolerance virologically suppressed to less than 400 copies/mL for at least 3 months on enfuvirtide-containing regimens either to continue the current regimen $(n=85)$, or to switch enfuvirtide to raltegravir $(n=85) .{ }^{28}$ The study was undertaken due to the high incidence of patient dissatisfaction among patients on enfuvirtide, owing primarily to injection-site reactions and the inconvenience of twice-daily subcutaneous injections. At 24 weeks, similar numbers of patients in each arm had achieved HIV-1 RNA levels of less than 50 copies/mL: 88\% in the enfuvirtide arm, 89\% in the raltegravir arm. One patient in each arm experienced virologic failure, and there were no significant changes in either arm in CD4+ cell counts through 24 weeks.

The SWITCHMRK ${ }^{29}$ trials examined a switch from a suppressive lopinavir/ritonavir-based regimen in treatment-experienced patients to a raltegravir-based regimen in terms of maintenance of viral suppression as well as lipid profiles. Although lopinavir/ritonavir is a potent PI with well-documented efficacy and durability, it is associated with significant elevations in serum lipid levels. Protocols 032 and 033 are parallel, randomized, multinational, double-blind trials. The approximately 350 patients enrolled in each 
protocol were required to have undetectable HIV-RNA levels for at least 3 months on a lopinavir/ritonavir-based regimen containing at least 2 NRTIs. Lipid-lowering therapy in the 12 weeks preceding study entry was not permitted. Of note, patients in both arms of each protocol had extensive treatment experience (median duration of previous ART 3.3 years in the raltegravir arm and 3.6 years in the lopinavir/ritonavir arm in Protocol 032; 3.7 vs 4.6 years, respectively, in Protocol 033). For the primary endpoint of HIV-1 RNA less than 50 copies $/ \mathrm{mL}$, noninferiority criteria were pre-specified as a lower limit of the $95 \%$ confidence interval (CI) of less than or equal to $12 \%$. Mean changes in lipid parameters were analyzed at week 12 . In both SWITCHMRK 1 and 2 , patients in the raltegravir arms less often maintained HIV-1 RNA levels of less than 50 copies/mL (81 vs $87 \%$ in Protocol 032; 88 vs 94\% in Protocol 033), and thus did not meet noninferiority criteria for viral suppression. However, there were significant improvements in lipids at week 12 in the raltegravir arms, with fasting total cholesterol, nonHDL cholesterol, and triglycerides statistically significantly lower in raltegravir arms. Pooled data from the parallel trials were examined to investigate further the increased rates of virologic failure in patients switched to raltegravir: failure was confirmed in 32 patients on raltegravir and in 17 patients on lopinavir/ritonavir. Resistance analysis was available for 10 patients from SWITCHMRK 2, two of whom failed raltegravir. Genotypic analysis revealed 7 of these patients harbored integrase mutations at the time of virologic failure, including 5 patients with the $\mathrm{N} 155 \mathrm{H}, 5$ with the Q148R/H, and 2 with the Y143C/S. Four of these patients also harbored multiple reverse transcriptase mutations, all had the M184V. While definitive explanations for these disparities are still elusive, it is likely that this treatment-experienced group of patients who had achieved viral suppression on lopinavir/ ritonavir harbored some degree of NRTI resistance at study entry, thereby increasing the theoretical risk of failure. Raltegravir performs best when combined with other, presumably fully active agents. This will likely occur more often with newer medications. Investigators examined the incidence of virologic failure in 143 heavily treatment experienced patients receiving raltegravir for the first time in combination with other agents, and found that rates of failure were lowest with raltegravir-containing regimens that included darunavir/ritonavir, maraviroc, enfuvirtide, or etravirine (2\%). Twenty-six percent of patients whose regimens did not include one of these agents experienced failure $(P<0.001) .{ }^{30}$ The ANRS 139 TRIO trial ${ }^{31}$ examined the use of raltegravir, etravirine and ritonavir-boosted darunavir for the treatment of patients with multidrug-resistant virus. Patients enrolled were naïve to the three study drugs, and $87 \%$ also received OBT. At week $48,86 \%$ (95\% CI $80 \%$ to $93 \%$ ) of patients had an HIV RNA level of less than 50 copies $/ \mathrm{mL}$, and the median increase in CD4 cell count was 108 cells $/ \mathrm{mm}^{3}$. These recent data underscore the important concept of combining raltegravir with other active agents, in order to maximize its efficacy and durability.

\section{Safety and tolerability}

Raltegravir is generally very well tolerated, and its safety in different patient populations has been demonstrated repeatedly in clinical trials. Initial Phase I studies that tested varying doses of the drug confirmed safety of raltegravir monotherapy at doses up to $1600 \mathrm{mg}$ daily for 10 days, and there were no drug-related discontinuations. ${ }^{15}$ The most common drug-related side effects reported were headache and fatigue characterized as mild to moderate in intensity. The most recent results from the largest clinical trials involving raltegravir administration to treatment-naïve and -experienced patients, STARTMRK and BENCHMRK, respectively, confirm its safety and tolerability. ${ }^{18,22}$

Most of the 566 patients enrolled in the STARTMRK ${ }^{18}$ trials experienced at least one clinical adverse event (AE), but more were receiving efavirenz (95.6\% vs $90 \%$ on raltegravir). Importantly, clinical AEs judged by investigators to be drug-related were significantly greater in efavirenz arms (77\% vs $44 \%$ in raltegravir arms, $P<0.001$ ). There were no significant differences in rates of serious AEs or AEs leading to study discontinuation between groups. Specific AEs occurring in more than $10 \%$ of patients on either drug were dizziness, headache, and abnormal dreams: all were more common in patients receiving efavirenz. Significantly more patients on efavirenz had experienced neuropsychiatric symptoms by week 8 ( $52 \%$ vs $20 \%$ on raltegravir, $P<0.001)$. At least $2 \%$ of patients in either arm experienced the following laboratory-associated AEs: elevated aspartate aminotransferase and elevated alanine aminotransferase. Grade 3 or 4 laboratory-associated AEs were generally uncommon, and occurred with similar frequency in each of the study arms.

Ninety-six week data from the BENCHMRK trials ${ }^{22}$ yielded similar results with regard to raltegravir safety and tolerability in treatment-experienced patients on OBR. Thirty-three percent of the 462 patients on raltegravir plus OBT vs $52 \%$ of patients on placebo plus OBT experienced a drug-related $\mathrm{AE}(P=\mathrm{NS})$, and $3.7 \%$ of patients on raltegravir discontinued drug due to an AE (vs $5.1 \%$ on placebo). 
The most common clinical AEs were diarrhea, nausea, and headache in both groups: only fatigue and abdominal distension were more common in raltegravir arms. Grade 3 or 4 laboratory abnormalities were equally uncommon in each arm; grade 4 elevations of creatine kinase ( $\geq 20.0$ times the upper limit of normal) occurred in $3 \%$ of patients receiving raltegravir and $0.8 \%$ of patients on placebo. Cancer events were similar in frequency as well: $3 \%$ vs $2.6 \%$ in raltegravir and placebo groups, respectively, yielding a relative risk of 1.1 (95\% CI 0.5, 3.1). Further analysis including data from all double-blind Phase II and III studies resulted in malignancy rates of 1.7/100 PYR for raltegravir, and 2.2/100 PYR for comparator groups (relative risk of $0.8,95 \% \mathrm{CI} 0.4,1.5){ }^{32}$

\section{Conclusions}

Raltegravir, the first INSTI, is currently approved for the treatment of HIV-1 infection in patients naïve to antiretroviral therapy, and heavily treatment-experienced. It retains activity against virus with known reverse transcriptase and protease mutations and both CCR5- and CXCR4-tropic virus. To date there is little known transmitted integrase resistance. Raltegravir has been proven non-inferior to standard recommended regimens in terms of virologic and immunologic efficacy, as well as safety and tolerability, and may in fact be better tolerated than most alternative regimens. Specifically, raltegravir has more favorable effects on lipids than comparator drugs efavirenz and lopinavir/ritonavir, and to that end may be a reasonable option for patients with hyperlipidemia and other cardiovascular risk factors. In an aging HIV-infected patient population with accelerated metabolic and vascular complications, raltegravir may prove particularly useful. Although available data from switch studies has been somewhat promising, there is ongoing concern with regard to the relatively low resistance barrier and potential for failure on raltegravir-based regimens that lack at least one other active agent. Results from controlled, blinded switch studies are forthcoming and will be important in considering these treatment strategies.

Raltegravir may find its place in other treatment situations in the near future, given its rapid and profound viral suppression. Potential areas of investigation include its use for post-exposure prophylaxis, acute infection, and prevention of perinatal infection. We may also see increasing raltegravir use in the context of class-sparing strategies, a concept also under current investigation.

In summary, the first integrase inhibitor has been a timely addition to our armamentarium of antiretroviral therapies. It has proven highly effective in patients with multidrug-resistant
HIV-1 infection, and is safe and effective in newly diagnosed patients. Results from ongoing investigations of raltegravir use in novel treatment strategies and patient populations will help determine the breadth of its use as the epidemic continues to affect an aging HIV-infected population.

\section{Disclosures}

Dr Steigbigel receives research support from Merck Research Laboratories, Boehringer Ingelheim, Bristol-Myers Squibb, and NexBio Pharmaceuticals. He is on the speakers bureau for Merck, as well as the advisory board for Boehringer Ingelheim. Dr Chirch has no conflicts of interest to declare.

\section{References}

1. Panel on Antiretroviral Guidelines for Adults and Adolescents. Guidelines for the use of antiretroviral agents in HIV-1-infected adults and adolescents. Department of Health and Human Services. December 1, 2009;1-161. http://www.aidsinfo.nih.gov/ContentFiles/ AdultandAdolescentGL.pdf. Accessed 2009 December 20.

2. El-Sadr WM, Lundgren 2 JD, Neaton JD, et al. CD4+ count-guided interruption of antiretroviral treatment. N Engl J Med. 2006;355(22): 2283-2296.

3. Kitahata MM, Gange SJ, Abraham AG, et al. Effect of early versus deferred antiretroviral therapy for HIV on survival. $N$ Engl J Med. 2009;360(18):1815-1826.

4. Hirsch MS, Gunthard HF, Schapiro JM, et al. Antiretroviral drug resistance testing in adult HIV-1 infection: 2008 recommendations of an International AIDS Society-USA panel. Clin Infect Dis. 2008;47(2):266-285.

5. Cooper DA, Steigbigel RT, Gatell JM, et al. Subgroup and resistance analyses of raltegravir for resistant HIV-1 infection. $N$ Engl $J$ Med. 2008;359(4):355-365.

6. Chirch LM, Morrison S, Steigbigel RT; Treatment of HIV infection with raltegravir. Expert Opin Pharmacother. 2009;10(7):1203-1211.

7. Delelis O, Carayon K, Saib A, Deprez E, Mouscadet JF. Integrase and integration: biochemical activities of HIV-1 integrase. Retrovirology. 2008;5:114.

8. Espeseth AS, Felock P, Wolfe A, et al. HIV-1 integrase inhibitors that compete with the target DNA substrate define a unique strand transfer conformation for integrase. Proc Natl Acad Sci U S A. 2000;97(21):11244-11249.

9. Iwamoto M, Wenning LA, Petry AS, et al. Safety, tolerability, and pharmacokinetics of raltegravir after single and multiple doses in healthy subjects. Clin Pharmacol Ther. 2008;83(2):293-299.

10. Wenning LA, Hanley WD, Brainard DM, et al. Effect of rifampin, a potent inducer of drug-metabolizing enzymes, on the pharmacokinetics of raltegravir. Antimicrob Agents Chemother. 2009;53(7):2852-2856.

11. Hanley WD, Wenning LA, Moreau A, et al. Effect of tipranavir-ritonavir on pharmacokinetics of raltegravir. Antimicrob Agents Chemother. 2009;53(7):2752-2755.

12. Iwamoto M, Wenning LA, Mistry GC, et al. Atazanavir modestly increases plasma levels of raltegravir in healthy subjects. Clin Infect Dis. 2008;47(1):137-140.

13. Iwamoto M, Wenning LA, Nguyen BY, et al. Effects of omeprazole on plasma levels of raltegravir. Clin Infect Dis. 2009;48(4):489-492.

14. Iwamoto M, Hanley WD, Petry AS, et al. Lack of a clinically important effect of moderate hepatic insufficiency and severe renal insufficiency on raltegravir pharmacokinetics. Antimicrob Agents Chemother. 2009;53(5):1747-1752. 
15. Markowitz M, Morales-Ramirez JO, Nguyen BY, et al. Antiretroviral activity, pharmacokinetics, and tolerability of MK-0518, a novel inhibitor of HIV-1 integrase, dosed as monotherapy for 10 days in treatment-naive HIV-1-infected individuals. J Acquir Immune Defic Syndr. 2006;43(5):509-515.

16. Markowitz M, Nguyen BY, Gotuzzo E, et al. Rapid and durable antiretroviral effect of the HIV-1 Integrase inhibitor raltegravir as part of combination therapy in treatment-naive patients with HIV-1 infection: results of a 48-week controlled study. J Acquir Immune Defic Syndr. 2007;46(2):125-133.

17. Markowitz M, Nguyen BY, Gotuzzo E, et al. Sustained Antiretroviral Effect of Raltegravir After 96 Weeks of Combination Therapy in Treatment-Naive Patients With HIV-1 Infection. J Acquir Immune Defic Syndr. 2009;52(3):350-356.

18. Lennox JL, DeJesus E, Lazzarin A, et al. Safety and efficacy of raltegravir-based versus efavirenz-based combination therapy in treatment-naive patients with HIV-1 infection: a multicentre, double-blind randomised controlled trial. Lancet. 2009;374(9692):796-806.

19. Sedaghat AR, Dinoso JB, Shen L, et al. Decay dynamics of HIV-1 depend on the inhibited stages of the viral life cycle. Proc Natl Acad Sci US A. 2008;105(12):4832-4837.

20. Grinsztejn B, Nguyen BY, Katlama C, et al. Safety and efficacy of the HIV-1 integrase inhibitor raltegravir (MK-0518) in treatment-experienced patients with multidrug-resistant virus: a phase II randomised controlled trial. Lancet. 2007;369(9569):1261-1269.

21. Steigbigel RT, Cooper DA, Kumar PN, et al. Raltegravir with optimized background therapy for resistant HIV-1 infection. $N$ Engl J Med 2008;359(4):339-354.

22. Steigbigel R, Cooper DA, Eron JE, et al. 96-week Results from BENCHMRK 1\&2, Phase III Studies of Raltegravir in Patients failing Antiretroviral Therapy with Triple-Class Resistant HIV. Conference on Retroviruses and Opportunistic Infections. Montreal, Canada; 2009.

23. Eron J, Nguyen BY, Steigbigel RT, et al. AIDS-Defining Conditions in the BENCHMRK 1 and 2 trials: 48 week analysis. 48th Annual ICAAC/IDSA 46th Annual Meeting. Washington, DC; 2008.

24. Miller M, Danovich R, Fransen S, et al. Analysis of resistance to the HIV-1 integrase inhibitor raltegravir: results from BENCHMRK 1 and 2. 48th Annual ICAAC/IDSA 46th Annual Meeting. Washington, DC 2008.
25. Fransen S, Gupta S, Danovich R, et al. Loss of raltegravir susceptibility of HIV-1 is conferred by multiple non-overlapping genetic pathways. $J$ Virol. 2009;83(22):11440-11446.

26. Zuger A: Report from the XVI International AIDS Conference. ACTG 5142 compares class-sparing regimens in treatment-naive patients. AIDS Clin Care. 2006;18(11):98.

27. Ripamonti D, Maggiolo F, Bombana E, et al. Efficacy, safety, and tolerability of dual therapy with raltegravir and atazanavir in antiretroviral experienced patients. 5th International AIDS Society Conference on HIV Pathogenesis, Treatment and Prevention. Cape Town, South Africa; 2009.

28. DeCastro N, Braun J, Charreau I, et al. Switch from enfuvirtide to raltegravir in highly treatment-experienced HIV-1 infected patients: a randomized open-label non-inferiority trial, EASIER - ANRS138. Program and Abstracts of the 16th Conference on Retroviruses and Opportunistic Infections. Montreal, Canada; 2009.

29. Eron J, Andrade J, Zajdenberg R, et al. Switching from stable lopinavir/ ritonavir-based to raltegravir-based combination ART resulted in a superior lipid profile at week 12 but did not demonstrate non-inferior virologic efficacy at week 24. Program and Abstracts of the 16th Conference on Retroviruses and Opportunistic Infections. Montreal, Canada; 2009

30. Rhee SY, Horberg M, Follansbee S, et al. Virologic response to raltegravir salvage and maintenance therapy. 47th IDSA Annual Meeting. Philadelphia, PA, 2009.

31. Yazdanpanah Y, Fagard C, Descamps D, et al. High rate of virologic suppression with raltegravir plus etravirine and darunavir/ritonavir among treatment-experienced patients infected with multidrug-resistant HIV: results of the ANRS TRIO trial. Clin Inf Dis. 2009;49(9): 1441-1449.

32. Cooper DA, Steigbigel RT, Lennox J, et al. Review of cancer incidence in raltegravir clinical trials. 16th Conference on Retroviruses and Opportunistic Infections. Montreal, Canada; 2009.
Infection and Drug Resistance

\section{Publish your work in this journal}

Infection and Drug Resistance is an international, peer-reviewed openaccess journal that focuses on the optimal treatment of infection (bacterial, fungal and viral) and the development and institution of preventive strategies to minimize the development and spread of resistance. The journal is specifically concerned with the epidemiology of antibiotic

\section{Dovepress}

resistance and the mechanisms of resistance development and diffusion in both hospitals and the community. The manuscript management system is completely online and includes a very quick and fair peerreview system, which is all easy to use. Visit http://www.dovepress.com/ testimonials.php to read real quotes from published authors. 